Products and Services In Practice is provided to readers using text and images from the manufacturer, supplier or distributor and does not imply endorsement by $B D J$ In Practice. Normal and prudent research should be exercised before purchase or use of any product mentioned.

Please send product and services news through to David Westgarth, $B D J$ In Practice via: David.Westgarth@bda.org

\section{The innovative}

\section{new autoclave}

Eschmann has recently launched its new Little Sister SES 3020B autoclave - a versatile option for professionals who want to keep at the very cutting-edge of infection control.

An innovatively designed system that can comfortably fit on a standard work surface, the new autoclave features a large, 23-litre capacity chamber and can process up to $6 \mathrm{~kg}$ of instruments. Plus, due to its dedicated long instrument tray, it can process instruments up to $370 \mathrm{~mm}$ in length.

The system boasts a number of features making it a must-have for the modern professional, including the ability to switch between ' $\mathrm{N}$ ' type and 'B' type cycles to accommodate every type of load. Furthermore, inbuilt multiple processors guarantee the sterility of each cycle, keeping compliance at the forefront of your infection control process.

To find out more about the state-ofthe-art Little Sister SES 3020B, contact the team at Eschmann.

For more information on the highly effective and affordable range of decontamination equipment and products from Eschmann, visit www.eschmann. co.uk or call 01903875787

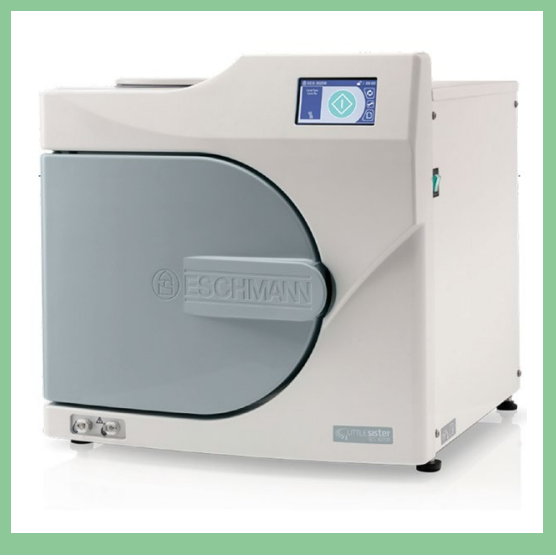

\title{
Dental practices can now recycle hard-to-recycle waste
}

As awareness of the issue of waste and single use plastics grows, consumers are increasingly looking to make more sustainable choices as they go about their daily lives. Of course, when it comes to oral care, a patient's health is the top priority, and unfortunately plastic waste is unavoidable.

Thankfully, TerraCycle's team of engineers, polymer scientists and recycling experts have over 20 years of experience in keeping otherwise unrecyclable materials in use and out of landfills and incineration, and have come up with a solution specifically for used oral care products.

The Oral Care Waste and Packaging Zero Waste Box is the first comprehensive recycling solution for hard-to-recycle oral care products and packaging, developed by the award-winning waste experts at TerraCycle.

Whether you're looking to recycle toothpaste tubes and caps, plastic toothbrushes, electric toothbrush heads which don't contain an RFID microchip or

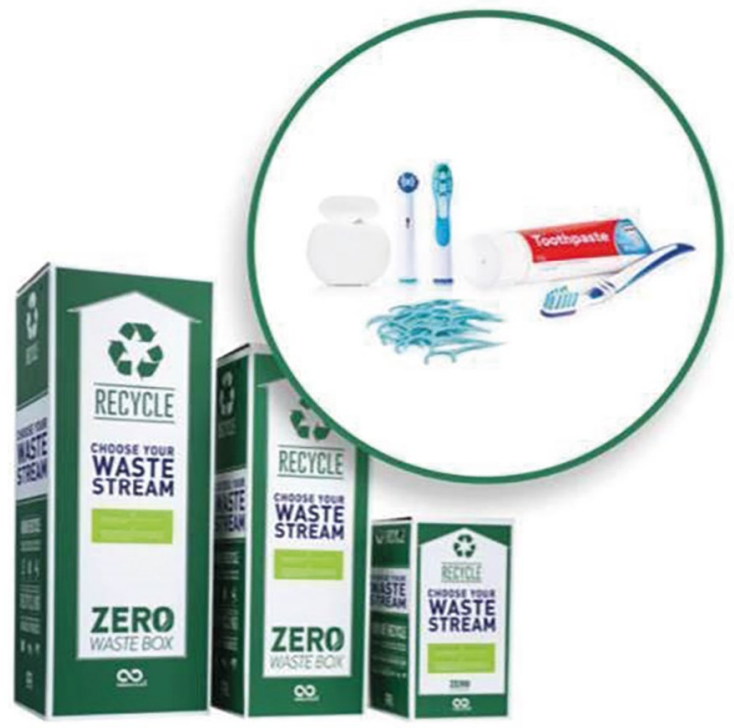

interdental brushes and floss containers, the Zero Waste Box system is an allinclusive recycling solution for all of those dental items that are typically considered non-recyclable through traditional council recycling systems.

The Zero Waste Box system is convenient and easy to use, making it the perfect option for dental practices looking to offset their impacts and reduce their environmental footprint. Aside from the obvious environmental benefits, an Oral Care Waste and Packaging Zero Waste Box also offers advantages such as bolstering a practice's reputation within the local community, singling them out as a positive changemaker for a more sustainable planet, generating potential positive word of mouth, increasing engagement with their existing patients, and even gaining new patients.

Zero Waste Boxes come in one of three sizes, small medium and large, with prices ranging from $£ 134.21$ for a small box, which will hold approximately 250 waste items, to $£ 263.71$ for a large box which will hold around 1,250 items. The price of each box includes the cost of TerraCycle sending it to the customer, the prepaid shipping label which the customer then uses to send their full box back to TerraCycle, and the cost of processing the waste.

For more

information visit www.terracycle.com/ en-GB. 